\title{
Structural Dynamics of Supported Metal Nanoparticles
}

\author{
Judith C. Yang ${ }^{1}$ \\ 1. Dept. of Chemical and Petroleum Engineering, Dept. of Physics, University of Pittsburgh, Pittsburgh, \\ PA (USA)
}

As more than $90 \%$ of chemical industries use catalytic materials, the resulting impact of catalysis on the global economy is estimated to exceed $\$ 10$ trillion per year.[1] Heterogeneous catalysis is also of substantial importance to energy generation, storage, sustainability and green chemistry. Yet technological advancements in catalysis have largely relied on costly and time-consuming trial-and-error approaches. It therefore remains a significant need in research to fundamentally understand and predict the local structure and stability of catalytic materials that can be specifically tailored by design and optimized for an application in technology. Our focus is on the development of integrated in situ and quantitative characterization and modeling tools and their applications appropriate for carrying out detailed studies on metallic nanoscale clusters. Complementary experimental work, such as environmental transmission electron microscopy (TEM) combined with synchrotron spectroscopy, is integrated with theoretical calculations for fundamental understandings of catalysis. It is now clear that the structural dynamics of small metallic clusters is actually quite complex.

For example, we have shown that the structures of Pt nanoparticles (NPs) may be both ordered and disordered, depending on the size, support and adsorbates. While bulk amorphous Pt is unstable, its existence in NPs is a manifestation of their mesoscopic nature.[2] Theoretical simulations show that the Pt NPs are not static, but show highly fluxional dynamics.

Furthermore, increasingly complex bimetallics are being synthesized to optimize catalytic reactions, yet their effectiveness depends on their structural integrity. We have also explored the environmental stability of bimetallic materials as a function of temperature and atmosphere to determine the range of environmental conditions where the NPs will be stable. For example, as NiCo bimetallic catalysts have shown beneficial activity for methane production, we examined core-shell reconfiguration of NiCo by X-ray photoelectron spectroscopy (XPS) and in situ (S)TEM. The reconfiguration was noted to occur in a stepwise process of surface oxide removal and metal segregation at temperatures above $300{ }^{\circ} \mathrm{C}$. These dynamic in situ studies provide mechanistic insights into the environmental stability of these nanoparticles, which is crucial for appropriate technological applications.

We are also interested in the development of quantitative STEM as it offers a way to extract the 3D structure of nanoparticles at the atomic scale, information that is crucial for interpreting ensembleaveraged data and as inputs for computational models. We are pursuing two approaches for expanding the capability and usability of quantitative STEM techniques. First, we have adapted it for use on a conventional (S)TEM, bringing it to a much broader field of instruments. Our high-throughput, semiautomated technique allows for atom-counting on statistically robust population sizes. Second, we are developing an approach that enables quantitative STEM to be performed on high-end analytic (S)TEMs equipped with cold-field emission guns (CFEG) through the use of real-time current calibration. [3]. Perspectives on the potential and limitations of quantitative and in situ electron microscopy for catalysis science will be presented. 
References:

[1] R. Shiju and V. V. Guliants, Appl. Catal., A, 356, (2009), p. 1-17.

[2] L. Li, L. Wang, D. Johnson, Z. Zhang, S. Sanchez, J. Kang, R.Nuzzo, Q. Wang, A. Frenkel, J. Li, J. Ciston, E. Stach, J. Yang, J. Am. Chem. Soc. 135, (2013), p. 13062-13072

[3] This work was supported by DOE BES through grant DE FG02-03ER15476 and NSF DMREF grant CHE-1534630. The electron microscopy work was performed using the facilities at the Center for Functional Nanomaterials at Brookhaven National Laboratory, which is supported by DOE BES through contract DE-SC0012704 and the National Center of Electron Microscopy, Lawrence Berkeley National Laboratory (DOE-BES: DE-AC02-05CH11231). The theoretical simulations were performed at Ames Laboratory which is operated by Iowa State University and supported by DOE-BES (DE-AC0207CH11358). 